\title{
Supporting Adherence to Highly Active Antiretroviral Therapy and Protected Sex Among People Living with HIV / AIDS: The Role of Patient-Provider Communication in Rio De Janeiro, Brazil
}

\author{
JESSICA FEHRINGER, M.H.S., ${ }^{1}$ FRANCISCO I. BASTOS, M.D., Ph.D., ${ }^{2}$ \\ ELIZE MASSARD, M.P.H., ${ }^{2}$ LEONARDO MAIA, M.D., ${ }^{3}$ J.H. PILOTTO, M.D., ${ }^{2,4}$ \\ and DEANNA KERRIGAN, Ph.D., M.P.H. ${ }^{1}$
}

\begin{abstract}
This exploratory study examined patient-provider communication dynamics regarding adherence to highly active antiretroviral therapy (HAART) and protective sexual behavior among people living with HIV/AIDS (PLWHA). We conducted 20 direct observations of routine consultations between PLWHA and care providers in two large public health clinics providing free HIV medications and clinical care to PLWHA in the greater Rio de Janeiro area of Brazil. Immediately after these observations, 20 semistructured in-depth interviews were conducted with observation participants regarding their communication with providers, overall clinic experience, and questions and concerns about adherence to HAART and safe sex. Findings from observations showed that patient-provider communication focused almost exclusively on biomedical aspects of HIV-related treatment such as symptom management. In most observations, adherence to HAART was addressed. However, questions posed by providers regarding adherence were generally close-ended and leading, discouraging an open exchange regarding potential difficulties related to adherence. HIV/sexually transmitted infection (STI)related protective behaviors were seldom addressed except when the patient displayed STI symptoms or was thought to be pregnant. In qualitative interviews, patients generally reported satisfaction with their providers, but also reported a variety of concerns and challenges related to adherence to HAART and protective sexual behavior that were not expressed in patient-provider interactions. We conclude that one way in which adherence to HAART and protective sexual behavior among PLWHA could be facilitated is by improving patient-provider communication on these topics, including increasing the frequency of openended, nonjudgmental dialogue initiated by care providers.
\end{abstract}

\footnotetext{
${ }^{1}$ Johns Hopkins Bloomberg School of Public Health, Baltimore, Maryland.

${ }^{2}$ Oswaldo Cruz Foundation, Rio de Janeiro, Brazil.

${ }^{3}$ Hospital PAM Ribeiro Neto, Rio de Janeiro, Brazil.

${ }^{4}$ Hospital Geral de Nova Iguaáu, Rio de Janeiro, Brazil.
} 


\section{INTRODUCTION}

$\mathbf{H}$ IGHLY ACTIVE ANTIRETROVIRAL THERAPY (HAART) has extended the survival and quality of life of many people living with HIV / AIDS (PLWHA) in a number of higher income countries where HAART has been publicly available since the mid-1990s. ${ }^{1-3}$ The survival of PLWHA living in Brazil has also increased dramatically since universal access to HAART was mandated by the federal government in $1996{ }^{4-7}$ However, in addition to these important gains, critical public health challenges related to the well-being of PLWHA as well as larger HIV / AIDS transmission dynamics remain, including how to better support adherence to HAART and protective sexual behaviors among PLWHA. Prior research on these two topics indicates that the adoption and maintenance of these desired public health outcomes is far from universal and is often quite challenging to achieve.

The possibility of both HIV treatment failure as well as the development of drug-resistant strains of HIV increase significantly when less than perfect adherence to HAART occurs. ${ }^{8}$ Several assessments of adherence to HAART conducted in higher income countries have found that up to $90 \%$ of patients report taking all their HAART medications over the last few days. However, when researchers inquire into adherence to HAART over longer periods of time, rates often drop considerably with more than a third of patients reporting missing doses over the last month. ${ }^{9}$ A recent study of adherence to HAART in Brazil, involving over 300 public health clinics and almost 2000 PLWHA, found that the prevalence of adherence to HAART, defined as taking $95 \%$ of HIV medicines over last 3 days, was $75 \% .{ }^{10}$ Adherence to HAART over longer periods of time has not yet been reported by the study team.

We also know that consistent protective sexual behavior among PLWHA continues to be a formidable challenge. A recent meta-analysis of available literature from the United States found that the reported rates of protective sex of PLWHA who were aware of their HIV status ranged from 60-87, depending on PLWHA subgroup, type of sexual partner and time frame. ${ }^{11}$ The rates of protected sex among
PLWHA in Brazil, based on the few studies conducted to date, are somewhat similar. ${ }^{12,13}$ For example, Paiva et al. ${ }^{12}$ found that $63 \%$ of 826 women living with HIV in Sao Paulo with steady male partners reported always using condoms with this partner type. While prior research indicates the need for continued prevention work among PLWHA and their sexual partners, it is also important to note that both studies stated that the reported rates of protected sex among PLWHA were significantly higher than the rates observed among those not infected with HIV within their respective study contexts. ${ }^{11,12}$

Factors found to be associated with adherence to HAART and safe sex, respectively, include some distinct as well as some overlapping elements. Adherence to HAART has been linked to both the treatment regime itself and the patient's experience with side effects. ${ }^{14}$ In addition to the biomedical and clinical issues specific to HAART, there are also several key sets of factors that appear to impact both adherence to HAART and safe sex among PLWHA, including the psychological state of mind of the individual patient as well as the social and material support systems to which a given PLWHA has access. ${ }^{9,15}$ One type of potential support system to which many PLWHA have ongoing access is the relationship with their clinical care providers.

Findings from higher income countries indicate that the relationship between clinical providers and their HIV-positive patients is an important predictor of adherence to HAART among PLWHA. ${ }^{16-19}$ Several different dimensions of the patient-provider relationship have been found to be associated with increased adherence to HAART. For example, Schneider et al. ${ }^{19}$ found that openness of communication, HIV-specific information, overall physician satisfaction, and physician trust were all independently associated with adherence to HAART.

Adherence to HAART is generally viewed as a routine aspect of the clinical care consultation for PLWHA. However, two different U.S.based studies regarding patient-provider communication and safe sex among PLWHA found that sex and sexuality are often not discussed within the context of ongoing care, with up to 
$25 \%$ of the PLWHA interviewed in these studies reporting they had never received safer sex counseling from their clinical care providers. ${ }^{20,21}$ Limited research has been conducted on the role of the patient-provider relationship and adherence to HAART or safer sexual behaviors among PLWHA in lower to middle-income income countries. In turn, this exploratory study sought to examine the dynamics of patient-provider communication and its relationship to adherence to HAART and safe sex among PLWHA in Rio de Janeiro, Brazil.

\section{MATERIALS AND METHODS}

\section{Study setting}

The study was conducted between September 2004 and March 2005 in two large government-run, public health centers in the greater Rio de Janeiro area of Brazil. Both centers provide free HIV-related care to generally lowerincome, PLWHA. The approximate number of PLWHA receiving ongoing care at these clinics was 1400 and 2000, respectively, with a total of approximately 1000 and 1400, respectively, receiving HAART per clinic. Each clinic had a total of approximately 12 clinical care providers (physicians, psychologists, social workers, and nurses) at the time of the study. Most providers were physicians, as the typical model of care at $\mathrm{HIV} / \mathrm{AIDS}$ clinics in Brazil is physician-driven, with very limited nursing staff or psychosocial care providers such as clinical psychologists or social workers. Each provider generally worked approximately 8 hours per day, consulting at least 10 patients during that time period. In each clinic, PLWHA receiving HAART generally came to the clinic once per month to receive their HIV medications and bi-monthly for follow-up clinical consultations. Physical examinations are generally carried out a given patient's initial consultation and in the case of specific symptoms or concerns, rather than in each consultation therein. Biomedical testing such as CD4 counts and viral load testing is generally conducted every 4-6 months per patient. It is important to note that all persons living with HIV meeting clinical criteria devel- oped by the Brazilian Ministry of Health are eligible to receive HAART in Brazil and that the government guarantees treatment to these persons (Brazilian Ministry of Health, Brazilian Legislation of STD and AIDS, Brasilia, Brasil, 2000). The clinical criteria for eligibility to receive HAART among PLWHA in Brazil state that antiretroviral therapy is recommended for all patients with HIV who are symptomatic and have a CD4 cell count below $350 / \mathrm{mm}^{3}$, and for asymptomatic patients with HIV with a CD4 cell count below 200/ $\mathrm{mm}^{3}$ (Brazilian Ministry of Health, National Program for STDs and AIDS, Recommendations for Anti-retroviral therapy in adults and adolescents infected with HIV, Brasilia, Brasil, 2004).

\section{Sample, recruitment, and participants}

This study utilized a cross-sectional design and purposive sampling to reach a subset of caregivers providing clinical and/or psychosocial care at the two participating study clinics and a group of 20 PLWHA to whom they provide ongoing care.

We recruited a total of eight care providers to participate in the study. Care providers met eligibility criteria if they provided ongoing, routine clinical and/or psychosocial care to HIV-positive patients receiving HAART. Provider participants were recruited with the assistance of the two clinic directors who informed the clinic's care providers of the study's objectives and encouraged their participation and then study staff approached the care providers to explain the purpose of the study in more detail and ask them if they were willing to participate. One care provider approached refused participation in the study citing a heavy workload as the reason for not participating. A private, formal informed consent process was undertaken with each of the other eight providers who expressed interest in participating.

Six of the participating providers were physicians providing ongoing clinical care to PLWHA (five females and one male; three per clinic) and two were social workers providing periodic psychosocial support to PLWHA (both female; one per clinic). Nurses and psychiatrists were not part of the sample because 
only one of the sites had these types of providers on staff and we wanted a similar provider composition across the sites. Both sites had a social worker providing psychosocial care to PLWHA. Only those persons who request to see social workers or those who are referred by their provider meet with social workers on an ongoing basis. While a minority of patients see social workers regularly, all patients meet with a social worker at some point during care.

We recruited a total of 20 PLWHA to participate in the study. We recruited at least 1 female and 1 male patient of each of the clinical care providers participating in the study. Patients met eligibility criteria if they: were HIV positive, on HAART, and had sexual intercourse within the last 3 months. In one clinic, eligible patients were called into a private office where the clinic's social worker gave a brief overview of the study and then asked if they would be willing to participate. If they were, the researcher then entered and the social worker left while the private consent process was then completed in this office. In the other clinic, the individual care providers asked eligible patients in their private offices if they were willing to participate without the presence of the researcher. If they were, the researcher then entered and the provider left while the consent process was completed in the office. We did not experience any patient participation refusals.

Ten women and 10 men living with HIV participated in the study, 8 from one clinic and 12 from the other. Three of the participants were homosexual men, 1 was a bisexual man, and the other 16 participants were heterosexual. The average age of participants was 37.3 years (range, 18-50) and the average educational level was 8.6 years, indicating that the majority had completed primary school (range, 5-15). The average length of time on HAART was 6.4 years (range, 5 months to 17 years). The large majority of participants reported having one regular sexual partner during the last 3 months $(17 / 20)$, with a range of $1-9$ partners.

\section{Data collection procedures}

Study methods consisted of 20 direct observations of scheduled patient-provider consul- tations, followed by semistructured, in-depth interviews with patients from these same consultations, immediately after their session. Observations of consultations lasted between 10 and 90 minutes, with an average of 29 minutes. These were guided by an observation guide that established areas of required observation. $\mathrm{Ob}-$ servation points included verbal and nonverbal communication, physical descriptions of the environment and descriptions of examinations conducted. The patient interview that immediately followed the observation lasted between 15 and 30 minutes, with an approximate average of 20 minutes, and was guided by a set of open-ended questions and subsequent probes. Patients were asked about their relationship with their provider, their experience at the clinic, concerns about protective sexual behavior and medication adherence, how any of the aforementioned could be improved, and interest in participating in group and/or individual support sessions. Both the direct observation guide and the interview guide were pilot tested six times in the HIV-clinic environment prior to study use. All direct observations and interviews were conducted in Portuguese by a local researcher trained in qualitative research methodology. The study protocol was approved the Institutional Review Boards (IRB) of the Johns Hopkins School of Public Health, the Oswaldo Cruz Foundation, and the National IRB of the Brazilian Ministry of Health.

\section{Data management and analysis}

Extensive field notes were taken during and after the observations and interviews and typed into computer files immediately following interactions with participants.

The interviewer/consult observer tried to capture verbatim conversation in her notes as frequently as possible. Observation and interview transcript files were coded and analyzed using a computer software program for text search, retrieval, and organization, ATLAS.ti 4.1 (ATLAS.ti Scientific Software Development $\mathrm{GmbH}$, Berlin, Germany). Codes were developed based on both interview and consult guides as well as based on themes emerging from the data collected using each methodology. 
The goal of the coding was to break up and then organize the data into categories that allowed for comparison of data both within and between categories. ${ }^{39}$ Following coding, we ran all data output corresponding to a given coding category and then reread and synthesized the data per category. We then developed memos for each category and synthesized the data across coding category developing a few salient themes which we would narrate in the paper. A final reading of the original transcripts in their entirety was carried out to contextualize the themes identified and assist in the articulation of study recommendations and conclusions.

\section{RESULTS}

\section{Overall clinical consultation dynamics}

Observation data indicated that patientprovider consultations generally focused on reviewing patient's test results for viral load levels, discussion of any symptoms the patient might be having, renewing prescriptions, scheduling for future tests and appointments, and updating demographic or contact information. Physical examinations were carried out in only three of the consultations. Two of these examinations were given when the patients exhibited symptoms (vision problems for one patient and feeling drugged after taking the evening HAART dose for the other patient) and the third was because the patient was pregnant. The general tone of consultations was relaxed and many involved some amount of joking and/or casual conversation between the provider and patient, with providers often asking questions about how the patient's family members were doing.

\section{Overall satisfaction with provider}

In in-depth interviews, most patients expressed satisfaction with their providers and could think of nothing negative about their relationship with their providers. Patients used a variety of positive descriptors for providers, such as "attentive," "concerned," "excellent," "patient," "cool," "very good," and "great." A number of patients said that they felt "com- fortable" with their providers and that they could talk about many things and "open up" with them. Several patients suggested that they had especially warm and friend or family-like relationships with their providers. "She raises my spirits . . . it is always good to have a person like that ... who leaves you well," related one male patient. A female participant stated, "It is as if she were another mother. She shares experiences with me... makes me an equal in what is going to happen . . . she's a great friend." Another female patient likewise described her social worker in the following manner, "She's the mother that I never had. We talk a lot ... When I'm embarrassed and can't talk to others she always welcomes me, she doesn't discriminate."

The few concerns reported by patients about their relationship with their providers involved issues of time. As one patient stated, "At times she's in a hurry because she has a lot of people to attend." This same patient later said that, "we wind up waiting a long time." Another participant explained that, "At times I don't open up with her ... I don't manage to ask a question. I prefer to keep it to myself . . . because of time ... the time is short."

\section{Openness of communication}

The majority of patients interviewed reported that they did not have any remaining doubts or concerns that they had not brought up with their provider during the consult. Statements such as "she always clears up everything" and "when I have a doubt, I ask" were common among participants. However, 5 of the 20 patients interviewed did state that they had lingering doubts or concerns that they did not articulate to their providers during the consultation prior to their interview. For example, several patients, when probed on the subject, said that they still had concerns over the use of condoms. The primary reasons given for not raising these concerns with their providers were that the subject was a "personal, intimate thing," and that "there are so many things that I forget [to ask]."

Other types of concerns expressed by participants in follow-up interviews related to both their own clinical care as well as the welfare of 
their family. For example, one patient did not understand "why some people manage to get tests and other don't." The patient before him was able to get a hemogram test done at the clinic but when he asked for this same test, he was told he needed to go offsite. Regarding the possibility of transmitting HIV to her children, another patient shared, "I have a doubt about my two children ... a at times they like to kiss people on the mouth . . . to eat off the same spoon. I don't know if they can." She said her reason for not asking her doctor about this was that she "didn't have the courage to ask," and felt "stupid," because she already saw on the television a "talk that said there was no problem using these things . . . but we are always preoccupied anyway."

Communication regarding adherence to HAART: "You're taking the medication correctly, right?"

In over half of the 20 patient-provider consultations observed, providers inquired about or discussed patients' adherence to HIV-related medications including HAART. Eight conversations were in the context of ongoing medication regimens and three were in the context of changing the patient's medication regimes. However, these interactions generally involved closed-ended and leading questioning patterns. For example, most providers asked patients at the beginning of the consult if they were taking their HAART medications "properly," "correctly," or simply if they were taking the medication at all. Patients typically responded, "yes" or "of course," with no further commentary. One physician asked a male patient, "You are taking it [the medication] correctly?" to which the patient responded, "Yes, sir." Another physician at the same clinic asked a female patient, "You are taking it [the medication] properly?" at which point the patient responded, "I don't even need a watch." A social worker at the other clinic similarly asked one patient, "You've been taking the medication correctly?" and the patient's response was, "I take it properly. I take Bactrim and another with a name I forget."

After a few questions regarding whether patients were "correctly" adhering to HAART, such as the examples mentioned above, pro- viders generally moved on to a different topic. In a few instances, providers asked more broadly if their patients "had any doubts?" related to their HAART medications. However, as the format of this question is also closedended, implying a yes or no response, it generally led to the patient saying nothing more than "no," before the conversation shifted to another topic.

In the remaining nine consultations adherence to HAART was not discussed at all. At times this appeared to be linked to providers' assumptions that adherence was going well based on seeing a low or undetectable viral load result from the patient's most recent test. For example, one provider simply stated to the patient, "You are taking the medication correctly. Its [viral load] been undetectable for a good period now." Additionally, in some of the consultations in which adherence was not discussed, it appeared that it may have been overlooked because of the patient's complaint of new symptoms which then became the focus of the consultation. For example, one patient felt that he had an inflammation on his penis or anus and the consultation focused on this; another had symptoms of lipodystrophy during menstruation as well as ophthalmologic symptoms. In one of the consultations in which medication adherence was not discussed, the patient had just been admitted as an inpatient, suggesting a potential prioritization of communication topics on the part of the physician.

There were a few exceptions to the brief, closed-ended style of adherence questioning described above. For example, when one patient mentioned to the social worker that she had just gone back to finish high school, the social worker asked, "How is it going taking your medicines and managing your courses?" The patient responded that she did not "see a barrier to taking the medicines," because she only had to take them "two times per day." The social worker continued, "and was it always easy?" to which the patient responded, "I never had a reaction. In the beginning I had trouble accepting [that she needed to take the meds]. I thought of myself . . . But, now I think of my children and that there is God in the sky and my medications in my purse (she laughs)." In a distinct observation involving a different provider 
another type of open-ended question was utilized to explore the issue of adherence. The provider asked, "How are you taking the medication?" When the patient responded, "Properly," the doctor further enquired, "How?" requiring the patient to provide more detail.

When asked about concerns regarding adherence to HAART in in-depth interviews, most participants reported that they did not find anything difficult about adhering to their medications. A few participants stated that they had problems with side effects or schedules "in the first days" or "before" but that these issues had been resolved and that now they were "normal" or "good." However, there were also several patients who told providers that adherence was going well, and then once questioned about potential difficulties in the postconsultation interview, shared a different story. One participant who had answered the provider during the consult that he was taking the medication "properly," explained to the interviewer afterwards that he had problems with the medication schedule because he does not eat at regular times. "At times I am late or early in taking it ... I get distracted and the time has passed." When the researcher asked how often this occurs, the patient responded, "It always happens. Today, no. Today I took it correctly ... At times when I'm watching TV, I get distracted. And at times I want to take it but I don't have food. I get depressed about this. Here in Rio I don't have family. Just me and God." Another patient similarly responded to his provider that he was taking the medication "properly." However, in his followup interview he revealed that sticking to the medication schedule was quite difficult and that he often took his medicines at least an hour after indicated.

In addition to challenges regarding take HAART medications on schedule, a few patients discussed problems with side effects or the difficulty of swallowing medications in postconsultation in-depth interviews. One participant shared, "I almost don't sleep. I take the medicine and I sleep, and when it's been about 4 hours I can't sleep anymore and I start walking around the house." Another patient described his concern that, "The pills are large and difficult to swallow ... so I have to take them with juice or with milk to help them go down." Neither of these two participant's providers asked them about medication adherence during the consultations observed.

When asked what the clinic or provider could do to help with medication adherence, patients generally said, "nothing," "I don't know," or "it's my thing." A few patients stated that they had already tried to discuss these issues with providers and did not receive much in the way of productive or supportive feedback. For example, one participant stated, "I already spoke with her [the doctor] and she said that I have to be more attentive and that I have to help myself."

\section{Patient-provider communication regarding protected sex or the lack thereof}

Overall, very few of the providers observed asked their patients about anything related to their sexual behavior. Of the 16 observations conducted with physicians, discussions of sexual behavior and/or condom use arose in only 3 cases. The first case occurred when a patient complained of burning urination and the following conversation was prompted:

\footnotetext{
Doctor: You use condoms in your sexual relations? Patient: I always use them.

Doctor: It could be the result of some abrasion from sexual activity then.

Patient: And I have had that. I definitely have.
}

The other example of a doctor asking about their patient's sexual behavior occurred when a woman who was recently diagnosed with HIV came to see her new provider. She was clearly pregnant and the doctor asked her about the "number of sexual partners" she had as well as whether or not she was using condoms. After the participant digressed off topic briefly, the doctor returned to the subject of condoms and challenged her, "Before the test you weren't in the habit of using them." The patient agreed and the doctor stated, "After you learned [HIV status], you started using them." The conversation then continues as follows:

Patient: After one condom broke we stopped using them.

Doctor: Why? 
Patient: Because he said it is already done [the virus passed between them].

Doctor: Ai ....Ai ...

Patient: But he told me he couldn't use them.

At the end of the consultation the doctor counseled the patient, "When you have relations without a condom you are subject to whatever problem the person has ... if you have already passed the virus to him, you could wind up exchanging the virus. A little passes and a little more will fill the pot. [He might say], 'Oh, it's already done' . . . But it's going to be done still more? This is not a justification. You have to use them."

Finally, one provider simply asked her patient, "Do you have condoms?" The patient started to respond, "If you had ... " but the doctor then interrupted and stated, "We have some kept here.", before the patient finished. The doctor then moved on to a different topic.

While issues related to protected sex were seldom discussed between physicians and their patients, in three of the four social worker consultations observed, safer sexual behavior was discussed. The following is an example of such an exchange:

Patient: It's complicated because many [sexual] partners don't want to [use condoms] . . . with the feminine [condoms], they don't know that I have it. Social worker: You put it in at the time or before? Patient: At the time. When he doesn't want [to use male condoms], I go in the bathroom and put it in. He doesn't feel it.

Social worker: You said that it is to avoid pregnancy? Is it easier to say that than to say to avoid disease?

Patient: It is.

Social worker: Pregnancy is scarier?

Patient: The disease doesn't come written on your forehead . . I I can go out with mine in my purse.

The two [male and female condoms].

Social worker: Most don't want to use?

Patient: They refuse.

In in-depth interviews following patientprovider observations, most patients did not express concerns about protective sexual behaviors and relayed the message that they knew they needed to use condoms. This sentiment was conveyed by one (female) patient when, referring to the need to use condoms, she said, "The instruction I have ... the knowledge .. . I will never do something wrong again."
Five of the 20 patients interviewed did expressed concerns regarding protected sex in follow-up interviews. Concerns were generally more prevalent among female participants regarding the difficulties they had trying to convince their male partners to use condoms. For example, one female participant stated, "A woman may be afraid to say no [to refuse sex without a condom], and this can jeopardize her." Another female shared, "One time we use it [a condom] and another time we don't . . I I know that it is not conscientious. But he wants to [have sex without a condom] and I give in."

Other concerns raised by patients regarding protective sexual behaviors involved the technical performance of condoms such as leakage or breakage as well as difficulties with themselves or their partners being able to enjoy sex with condoms. One participant discussed the problems she had had with the use of both male and female condoms and how this may cause problems in her health, "The male one started to give me allergies . . . and the female kind I can't manage to keep inside me ... you have to hold the condom . . . if you don't, it comes out . . I l love myself and without a condom, I don't have a chance. The love will end. I have an undetectable viral load and I take care of myself." On this same topic, another female participant shared her frustration that only male condoms were available at the clinic.

When asked what their provider or clinic could do to help with protective sexual behavior concerns, patients generally had no suggestions or described the problem as their own, one that the clinic could do nothing about because it is between them and their partner. One participant stated, "This is my thing alone." Likewise, another participant stated, "It is between he [her partner] and I."

\section{DISCUSSION}

Patients generally reported positive, friendly, and satisfying relationships with clinic providers. However, study findings indicate that there are potentially many missed or ineffectively utilized opportunities to address critical public health issues such as adherence to HAART and protected sex among PLWHA in ongoing clinical care consultations. 
While many providers in this study engaged in some level of communication regarding adherence to HAART with their patients, the form of communication was often closed-ended and leading. Rather than asking a patient if they are taking the medication "correctly," thereby limiting and discouraging open, in-depth communication, providers could ask, "How is your experience with your medication going lately?" as one social worker from the study inquired. While most patients did not report difficulties with adherence to HAART in in-depth interviews, some did report missing medication doses or taking medicines late due to problems with forgetfulness and/or diet, indicating the importance of more in-depth communication regarding such challenges. Most patients may understand and be able to follow the directions related to adhering to HAART, but as this and other research in Brazil has found, there are some patients who require special assistance. For example, Ceccato et al. ${ }^{22}$ found that $26.3 \%$ of patients in a sample of PLWHA attending public AIDS clinics in Brazil had insufficient understanding of their medication regimen, defined as responding accurately to $70 \%$ or less of key treatment-related questions on topics such as dosing, adverse reactions, and food requirements. The data also suggest that providers need to be better prepared to respond to patients who do express concerns regarding adherence to HAART. As the few of the patients interviewed who did report asking for help did not generally feel that their providers were interested in discussing their concerns or able to provide them with specific ideas or skills to assist them.

Most providers observed did not communicate at all with their patients about protective sexual behavior. The few exceptions to this were when patients exhibited obvious signs of lack of protective sexual behavior, such symptoms of a sexually transmitted infection or being pregnant. In follow-up interviews, most patients reported no problems with protective sexual behavior. Yet again, there were some participants that did report having concerns or problems that were going unaddressed. Concerns regarding safe sex were more prevalent among female participants as were reports that they were unable to convince their male part- ners to use condoms or having difficulties using specific types of condoms. The few women who did try to discuss such issues with their providers did not feel as if their questions and concerns were received without judgment or that their providers were interested in or prepared to give them advice on this subject.

In addition to the patients feeling judged in several instances by their providers, patients themselves used self-judging language at times regarding their struggles to maintain protective sexual behaviors and to adhere to HAART. For example, when discussing her inability to practice safer sex in the past, one female patient reported, "I will never do something wrong again." Another female participant who reported being unable to convince her partners to use condoms reflected on how her behavior was not "conscientious." Such statements suggest that in addition to access to accurate information regarding adherence or safe sex, deeper psychosocial issues such as internalized stigma and self-acceptance may be impacting both the quality of life as well as the health-related behaviors of PLWHA and should be contemplated within future intervention research regarding patient-provider communication among PLWHA.

One way to improve patient-provider communication is to implement continuing education and training for the care providers. In fact, a recent qualitative study in Brazil suggests that many physicians do not engage in adherence discussions precisely because they do not feel prepared. ${ }^{23}$ Yet, research regarding provider training to improve communication between care providers and PLWHA regarding safer sex has been linked to positive behavioral changes among PLWHA. For example, Richardson et al. ${ }^{24}$ saw significant reductions in HIV sexual risk behaviors among PLWHA with multiple partners within the context of a safer sex counseling intervention initiated by physicians in the United States. Clinics have also reinforced provider communication trainings, by developing written procedures and guidelines regarding the frequency of and approach to communication on topics such as safer sex. For example, Myers et $\mathrm{al}^{25}$ found that in clinics in the United States with written procedures on safer sex 
counseling, there was an increased probability that providers counseled HIV positive patients on safer sex issues.

Most of the interventions implemented in clinical care settings related to adherence to HAART documented in the literature tend to utilize clinic staff other than physicians, such as nurses, to engage patients in cognitive behavioral sessions outside of routine clinical or psychosocial care interactions. ${ }^{27}$ Such interventions are clearly important and needed. However, also of importance is the need for improved ongoing, routine patient-provider communication related to adherence to HAART, including communication between physicians and patients. We did not find any examples of interventions related to improving routine provider communication on HAART with physicians in the literature. Yet, the reality of the health care model in most middle to lower income countries is that it is physiciandriven, and often lacking in nonphysician staff such as nurses and psychosocial care providers. It is important to note when considering the possibility of encouraging increased, openended communication between providers and patients, that two studies in The Netherlands, one with gynecologists and the other with pediatricians, have looked at the length of the visit and found that improving physician communication did not lead to longer visit times, a potential barrier in the busy clinic environments where providers must see many patients on tight schedules, as is the case at the clinics from this study. ${ }^{28,29}$

The interventions that have been implemented to date regarding patient-provider communication in the context of ongoing care with PLWHA have been limited. Additionally, most have focused on the provision of information, education and/or counseling regarding topics such as safer sexual behaviors. Our study does indicate that enhanced discussion on safer sex is warranted. However, our findings also suggest that directive communication that works from the assumption that patients "should" be adhering to HAART or "should" be practicing safer sex, may further reinforce patient's anxiety, perceived sense of inadequacy and self-judgment, in the case that they are currently not implementing these behaviors as recommended by clinic staff. By engaging in a more open, nonjudgmental dialogue physicians, can slowly become more aware of the fears and insecurities with which their patients are struggling and reflect on ways that they may be able to assist them as possible or refer them to complementary psychosocial services if needed and available. Additionally, our findings indicate that there may be some patients with specific material constraints to adherence to HAART, such as the lack of adequate food, which must also be contemplated in future intervention research, particularly in lower income settings. A brief food security assessment tool could be developed and implemented periodically by care providers with patients, not only to ensure adherence to HAART, but to increase overall health and well-being.

This study provides important data regarding patient-provider communication surrounding two critical public health challenges: adherence to HAART and safer sex among PLWHA. However, this study represents initial work on this topic in the Brazilian context and in turn there are several limitations to the study that are important to note. Our small sample size of both providers and patients limits our ability to generalize our results as does our emphasis on specialized HIV / AIDS clinics in a cosmopolitan, urban area. The use of only one observation and interview per patient-provider pair limits our ability to draw conclusions about the more nuanced nature of each patient-provider dynamic. While we did attempt to establish interrater reliability by having a second researcher present during three of the pilot interviews, this researcher was not present throughout all of the data collection because of the potential added bias of multiple observers on the intimate patient-provider dynamic. Without this second perspective, we cannot be confident that another observer/interviewer would have collected similar data. Additionally, we receive feedback from one of the patient's interviewed who felt his provider was not normally as detailed as he was during the observed consultation. Despite these limitations, the data presented herein help to shed light on areas of patient-provider communication, which if improved, may lead to increased adherence to HAART, safer sexual behavior, and overall well-being among PLWHA. 


\section{ACKNOWLEDGMENTS}

We would like to thank all of the patients and providers who participated in this study. We would also like to recognize the contributions of Rosa Maria Pinheiro Rezende and the staff at Hospital PAM Ribeiro Neto and Hospital Geral de Nova Iguaçu. This research was supported by a Pilot Grant from the Johns Hopkins University Center for AIDS Research (CFAR), Grant P30 AI42855, as well as a Career Development Grant from the National Institute of Mental Health of the National Institutes of Health, K01 MH3491.

\section{REFERENCES}

1. Hogg RS, Heath KV, Yip B, et al. Improved survival among HIV-infected individuals following initiation of antiretroviral therapy. JAMA 1998;279:450-454.

2. Palella FJ Jr, Delaney KM, Moorman AC, et al. Declining morbidity and mortality among patients with advanced human immunodeficiency virus infection. HIV Outpatient Study Investigators. N Engl J Med 1998;338:853-860.

3. Detels R, Munoz A, McFarlane G, et al. Effectiveness of potent antiretroviral therapy on time to AIDS and death in men with know HIV infection duration. JAMA 1998;280:1497-1503.

4. Marins JR, Jamal LF, Chen SY, et al. Dramatic improvement in survival among adult Brazilian AIDS patients. AIDS 2003;17:1675-1682.

5. Teixeira PR, Vitoria MA, Barcarolo J. Antiretroviral treatment in resource-poor settings: the Brazilian experience. AIDS 200418(Suppl 3):S5-7.

6. Casseb J, Fonseca LA, Veiga AP, et al. AIDS incidence and mortality in a hospital-based cohort of HIV-1seropositive patients receiving highly active antiretroviral therapy in Sao Paulo, Brazil. AIDS Patient Care STDs 2003;17:447-452.

7. Guerreiro MF, Kerr-Pontes LR, Mota RS, et al. Survival of adult AIDS patients in a reference hospital of a metropolitan area in Brazil. Rev Saude Publica 2002;36:278-284.

8. Reynolds NR. Adherence to antiretroviral therapies: state of the science. Curr HIV Res 2004;2:207-214.

9. Ickovics JR, Meade CS. Adherence to HAART among patients with HIV: Breakthroughs and barriers. AIDS Care 2002;14:309-318.

10. Nemes MI, Carvalho HB, Souza MF. Antiretroviral therapy adherence in Brazil. AIDS 2004;18(Suppl 3):S15-20.

11. Marks G, Crepaz N, Senterfitt JW, Janssen RS. Metaanalysis of high-risk sexual behavior in persons aware and unaware they are infected with HIV in the United
States: Implications for HIV prevention programs. J Acquir Immune Defic Syndr 2005;39:446-453.

12. Paiva V, Latorre Mdo R, Gravato N, et al. Sexuality of women living with HIV/AIDS in Sao Paulo. Cad Saude Publica 2002;18:1609-1620.

13. Guimaraes MD, Boschi-Pinto C, Castilho EA. Safe sexual behaviour among female partners of HIV-infected men in Rio de Janeiro, Brazil. Int J STD AIDS 2001;12:334-341.

14. Chesney MA. Factors affecting adherence to antiretroviral therapy. Clin Infect Dis 2000;30(Suppl 2):S171-176.

15. Crepaz N, Marks G. Towards an understanding of sexual risk behavior in people living with HIV: a review of social, psychological and medical findings. AIDS 2002;16:135-149.

16. Preau M, Leport C, Salmon-Ceron D, et al. Health-related quality of life and patient-provider relationships in HIV-infected patients during the first three years after starting PI-containing antiretroviral treatment. AIDS Care 2004;16:649-661.

17. Murphy DA, Marelich WD, Hoffman D, Steers WN. Predictors of antiretroviral adherence. AIDS Care 2004;16:471-484.

18. Abel E, Painter L. Factors that influence adherence to HIV medications: Perceptions of women and health care providers. J Assoc Nurs AIDS Care 2003;14: 61-69.

19. Schneider J, Kaplan SH, Greenfield S, et al. Better physician-patient relationships are associated with higher reported adherence to antiretroviral therapy in patients with HIV infection. J Gen Intern Med 2004;19:1096-1103.

20. Marks G, Richardson JL, Crepaz N, et al. Are HIV care providers talking with patients about safer sex and disclosure?: A multi-clinic assessment. AIDS 2002;16: 1953-1957

21. Margolis AD, Wolitski RJ, Parsons JT, et al. Are healthcare providers talking to HIV-seropositive patients about safer sex? AIDS 2001;15:2335-2337.

22. Ceccato MG, Acurcio FA, Bonolo Pde F, Rocha GM, Guimaraes MD. HIV patients' understanding of information on antiretroviral therapy. Cad Saude Publica 2004;20:1388-1397.

23. Malta M, Petersen ML, Clair S, Freitas F, Bastos FI. Adherence to antiretroviral therapy: a qualitative study with physicians from Rio de Janeiro, Brazil. Cad Saude Publica 2005;21:1424-1432.

24. Richardson JL, Milam J, McCutchan A, et al. Effect of brief safer-sex counseling by medical providers to HIV-1 seropositive patients: a multi-clinic assessment. AIDS 2004;18:1179-1186.

25. Myers JJ, Steward WT, Charlebois E, Koester KA, Maiorana A, Morin SF. Written clinic procedures enhance delivery of HIV "prevention with positives" counseling in primary health care settings. J Acquir Immune Defic Syndr 2004;37(Suppl 2): S95-S100.

26. Simoni JM, Frick PA, Pantalone DW, Turner BJ. Antiretroviral adherence interventions: A review of cur- 
rent literature and ongoing studies. Top HIV Med 2003;11:185-198.

27. Van Dulmen AM, Holl RA. Effects of continuing paediatric education in interpersonal communication skills. Eur J Pediatr 2000;159:489-495.

28. Van Dulmen AM, van Weert JCM. Effects of gynaecological education on interpersonal communication skills. Br J Obstet Gynaecol 2001;108:485-491.
Address reprint requests to:

Deanna Kerrigan

Johns Hopkins Bloomberg School of Public Health 615 North Wolfe Street, E 5523A

Baltimore, MD 21205

E-mail: dkerriga@jhsph.edu 
Copyright of AIDS Patient Care \& STDs is the property of Mary Ann Liebert, Inc. and its content may not be copied or emailed to multiple sites or posted to a listserv without the copyright holder's express written permission. However, users may print, download, or email articles for individual use. 